Canum 2000 : Actes du 32e Congrès national d'analyse numérique

A. Blouza, I. Danaila, P. Joly, S.M. Kaber, B. Lucquin, F. Murat \& M. Postel, Éditeurs

ESAIM: Proceedings, Vol. 11, 2002, 41-60

http://www.emath.fr/Maths/Proc/Vol.11/

(C)2002, Société de Mathématiques Appliquées et Industrielles, EDP Sciences

\title{
Recent developments in topology design of materials and mechanisms
}

\author{
Martin P. Bendsøe
}

\begin{abstract}
Résumé. Cet article présente certaines méthodes d'optimisation topologique des milieux continus et leur utilisation dans la conception de matériaux et mécanismes. Ceci comprend l'utilisation de l'optimisation topologique dans la conception de matériaux ayant des propriétés limites et l'introduction de critères de flambage. On s'intéresse aussi à la conception de matériaux composites isotropes mettant en oeuvre les schémas d'interpolation à la mode en optimisation topologique. Enfin on présente des exemples de conception de mécanismes flexibles.
\end{abstract}

Mots clés. Optimisation topologique, microstructures, mécanismes flexibles.

\begin{abstract}
This paper gives a brief introduction to some of the methods used in topology design of continuum structures and to their use for the design of materials and mechanisms. This encompasses the use of topology design in design of materials with extreme properties, and the introduction of buckling criteria in such design problems. A topic is also the design of isotropic composites which realize certain popular interpolation schemes used in topology design (making the dog bite its tail). Finally, examples of the design of flexible mechanisms will be discussed.
\end{abstract}

Key words. Topology design, microstructures, mechanisms.

AMS subject classification. 74P, 74Q, 74M, 65M, 49J

\section{Introduction}

The area of computational variable-topology shape design of continuum structures is presently dominated by methods which employ a material distribution approach for a fixed reference domain, in the spirit of the so-called 'homogenization method' for topology design ([7], [4]). That is, the geometric representation is like a grey-scale rendering of an image, i.e., a raster representation of the geometry on a fixed reference domain. The physics of the problem is also defined on this fixed reference domain, in analogy to fictitious domain methods for finite element analysis. The technique has its origins in theoretical studies of existence of solutions in variational problems, in particular shape optimization problems, and in studies in theoretical material science of variational bounds on material properties. The area is presently oriented towards applications, and one now sees a fairly widespread use of topology design in industry. 
In this short introduction to the field we outline some of the basic ideas and methods, referring to existing literature for comprehensive surveys (e.g., [8], [36], [9], [12]). Also, reference is mostly made to recent papers that include bibliographies useful for on overview of the area - the presentation thus does not present a complete historical perspective.

The basic setting of continuum topology optimization is a '0-1' integer design problem (generalized shape optimization). Thus a design specifies whether there is solid material or void at every point in the chosen design region, without any further restrictions on the shape. In general, this class of problems is ill-posed in the continuum setting (cf., [25], [23]). Well-posed problems can be obtained by using their relaxed versions which usually incorporates microstructure, that is, optimal designs with grey-scale (see, e.g., [2], [13]). Relaxation usually yields a set of continuously variable design fields to be optimized over a fixed domain, a convenient feature for computations (the integer $0-1$ format is avoided). The form of the relaxed setting is only known for a limited class of problems and much work is still needed in this area.

An alternative is to restrict the space of admissible solutions. This can typically be accomplished by enforcing a bound on the perimeter of the design (see [33], and references therein), by use of a filter limiting minimum scale (see [44] and [10] for an overview), or by imposing constraints on gradients of grey-scales defining geometry (see [34], and references therein). The problems with restricted design freedom are typically - by some compactness properties - wellposed in the setting of a continuum description of the design problem (existence still requires, for each case, a formal proof, as seen in [5], [10]). In a 0-1 setting, the restriction approach leads to 'classical designs' and the physical modelling is determined solely by the properties of the given solid material. The major challenge is the solution of a large-scale integer programming problem. The high cost of function calls for these problems typically precludes the use of genetic algorithms or simulated annealing impractical for large-scale problems - this is an area that should have more focus in the coming years (see, however, [6] for a recent successful application of Lagrangian duality to the large scale integer problem). Thus the typical approach is to replace the integer variables with continuous variables together with some form of penalty that steers the solution to discrete 0-1 values. A key part of these methods is the introduction of interpolation functions (often interpreted as material densities) that express various physical quantities (e.g., material stiffness, cost, etc.) as a function of the continuous variables. Moreover, geometric properties also require suitable interpretation. Often such interpolations can be obtained from the microstructures describing the relaxed formulations. It is important, however, to recognize that the ultimate goal remains that of obtaining a black-and-white design.

\section{Problem form}

In topology design we seek, for given choice of objective and constraint, the optimal distribution of material in a fixed reference domain $\Omega$ in $\mathbf{R}^{2}$ or $\mathbf{R}^{\mathbf{3}}$. Here we consider a mechanical element as a body occupying a domain $\Omega^{m} \subseteq \Omega$. Referring to the reference domain $\Omega$ on which applied loads and boundary conditions are defined, we can define an example 'control in the coefficients' 
problem, here for simplicity taken as the minimum compliance problem:

$$
\begin{aligned}
& \min _{C, u} \int_{\Omega} f u d \Omega, \quad \text { subject to: } \\
& \int_{\Omega} C_{i j k l}(x) \epsilon_{i j}(u)(x) \epsilon_{k l}(v)(x) d \Omega=\int_{\Omega} f v d \Omega \text { for all } v \in U \\
& C_{i j k l} \in E_{\mathrm{ad}}
\end{aligned}
$$

Here $U$ is the space of kinematically admissible displacement fields, $u$ the equilibrium displacement, $f$ the forces, and $\epsilon(u)$ linearized strains. The rigidity tensor $C_{i j k l}$ is the design variable of our problem. The definition of the set of admissible rigidity tensors is what distinguishes various settings for the design problem. A classical variant of problem (1) is the so-called variable thickness problem, where the set of admissible designs is defined through a thickness function $h$ :

$$
\begin{aligned}
& C_{i j k l}(x)=h(x) C_{i j k l}^{0} \quad h \in L^{\infty}(\Omega) \\
& \int_{\Omega} h d \Omega=V, \quad 0 \leq h_{\min } \leq h \leq h_{\max }
\end{aligned}
$$

for given material properties $C_{i j k l}^{0}$ and given volume $V$. This problem is rather special, with existence of solutions in the 'naive' setting (see [32] and references therein).

For a topology design where we work with domains $\Omega^{m}$ of material points, the admissible designs are defined by a point-wise volume fraction of a given material attaining the values zero or one (a black-and-white design):

$$
\begin{aligned}
& C_{i j k l}(x)=\Theta(x) C_{i j k l}^{0}, \\
& \Theta(x)=1, x \in \Omega^{m} ; \quad \Theta(x)=0, x \in \Omega \backslash \Omega^{m} \\
& \operatorname{Vol}\left(\Omega^{m}\right)=\int_{\Omega} \Theta(x) d \Omega=V
\end{aligned}
$$

Materials with a structural hierarchy often allow for stiffer structures, as seen in nature in bone, wood, etc. and used in composite structures (see Figure 1). Here this can lead to a lack of existence of solutions, as composites can be constructed as limiting sequences of designs defined by (3); however, composites are not covered by (3) (for $C_{i j k l}^{0}$ isotropic, the material in (3) is isotropic, but a composite can be anisotropic).
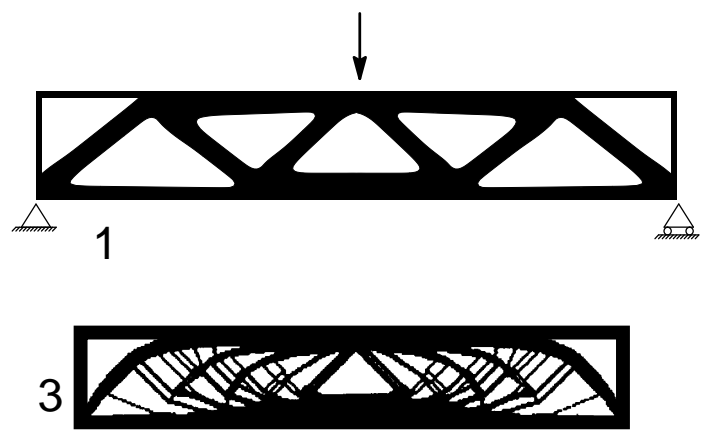

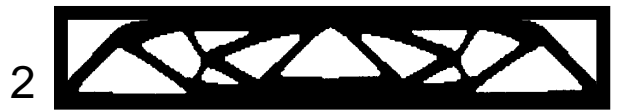

4

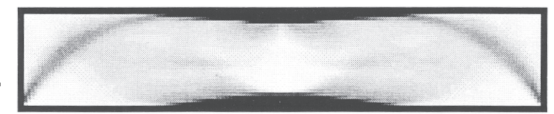

Figure 1: Elements of a minimizing sequence of designs with finer and finer scales, with the limit (4) consisting of composites in large areas. 


\section{Computations}

For computations a finite dimensional version of the problem (1) is generated first, normally with finite element approximations of displacement fields and design variables. The generic form of the resulting non-linear, non-convex, large scale optimization problem is

$$
\begin{aligned}
& \inf p^{T} x \\
& \quad A(d) x=p \\
& \quad \sum t_{i}=V, \quad 0<t_{\text {min }} \leq t_{i} \leq t_{\text {max }}
\end{aligned}
$$

Here $x$ denotes nodal displacements and $t$ the design variables, entering the equilibrium equation through the stiffness matrix $A$.

If this problem is solved directly via mathematical programming techniques one treats both displacements and design variables as independent variables and the equilibrium equation is solved by the optimization code. This is thus for even moderately sized problems normally only viable by use of specially developed algorthms that utilize the problem structure (an example of this can be found in [50]). In some cases, as for the minimum compliance case, it is possible to find explicit formulas for the optimal design variables in terms of the state variables. This allows for a reduction of the number of variables, and the resulting problem is an equilibirum problem with a non-smooth potential. For continuum design, such a technique, based on a stress based formulation, is used in, e.g., [4], [2].

The traditional approach is to rewrite the problem as a problem in the design variables only:

$$
\begin{aligned}
& \inf F(t) \\
& \sum t_{i}=V \\
& 0<t_{\text {min }} \leq t_{i} \leq t_{\text {max }}
\end{aligned}
$$

with the function:

$$
\begin{aligned}
& F(t)=p^{T} x \\
& \text { where } x \text { solves } A(t) x=p
\end{aligned}
$$

If the optimization algorithm to be used for solving (5) requires function gradients, sensitivity analysis (i.e., efficient means to compute the derivatives of functions involving the displacements) becomes a central subject. This usually involves the so-called adjoint system, as also known from control theory. Sensitivity analysis is an important area of computer aided design, as it provides information on changes in performance with respect to design variables, see for example papers in [21]. The main rationale behind using (5) as the basic format for computational optimization is the use of commercial software packages for finite element analysis and that it is fairly straightforward to generalize to a broad class of problems. Moreover, in such problems as shape design via CAD defined boundaries, the number of design variables is usually comparably small, making (5) a moderately sized problem (with 'expensive' function calls). For topology design, even problem (5) is large-scale; in many cases the key point to success is to formulate relevant problems involving only a moderate number of constraints, so that dual methods can be 
effective. Here techniques based on separable, convex approximations are particularly popular (CONLIN, see [16], and MMA, see [47]).

Finally, we note that the continuum problem at hand is a two field problem which may develop checkerboards in computations (as for a Stokes flow problem). For a fine enough mesh, geometric complexity constraints usually removes such effects, but in for example a relaxed setting special care must be taken to avoid such 'polluted' results (see [44] for an overview).

\section{Homogenized materials, relaxation, and material design}

Inspired by theoretical studies on generalized shape design in conduction and torsion problems and by numerical and theoretical work related to plate design (see, e.g., [11], [24], [19]), [26]) initial work on numerical methods for topology design of continuum structures was based on using composite materials as the basis for describing varying material properties in space ([7]). Composites consisting of square or rectangular holes in periodically repeated square cells were first used for planar problems and homogenization techniques were used for computing effective moduli of the materials. Hence the use of the phrase 'the homogenization method for topology design'. The method involves working with orthotropic or anisotropic materials. This adds to the requirements of the finite element analysis code, but the main additional complications is the extra design variables required to describe the structure. Thus, a microstructure with square holes in square cells require two distributed variables, as the material properties at each point of the structure will depend on the size-variable characterizing the hole size and on one variable characterizing the angle of rotation of the material axes.

The types of microstructures just mentioned do not provide for the full relaxation for the design problem, which in principle should encompass all composites formed by G-convergence (or H-convergence). However, for the compliance problem one only needs the composites which provides for the stiffest composites, defined in similar terms as the problems of bounds on effective material properties, as studied in the theory of composites (see [12] for a comprehensive bibliography). Here it has been proved that for example so-called ranked laminates (layers) are in fact optimal for stiffness problems (for an overview, see for example [3], [4]). Their effective properties can be given in analytical form (and in a subtle recursive form, cf., [17]), a central aspect for the existence proofs and for their use in computations. With layered materials, existence of solutions to the minimum compliance problem for both single and multiple load cases is obtained, without any need for additional constraints on the design space (e.g., without constraints on the geometric complexity), and thus interpolation (i.e., continuous design variables) and relaxation are both provided for. However, the relaxed models seldom result in black-and-white designs in themselves.

The construction of the relaxation can in esssence be seen as problem of design of composites. This can be seen by rewriting problem (1) in the form

$$
\max _{C \in E_{\mathrm{ad}}} \min _{v \in U} \int_{\Omega} C_{i j k l}(x) \epsilon_{i j}(v)(x) \epsilon_{k l}(v)(x) d \Omega-2 \int_{\Omega} f v d \Omega
$$

where the inner problem is the minimum potential energy problem, with value at equilibrium equal to the negative of compliance. By interchanging the 'max' and the 'min' and considering 
the pointwise optimal choice of rigidity tensor $C$, one identifies an auxiliary sub-problem that will generate a bound on performance:

$$
\max _{C} C_{i j k l}(x) \epsilon_{i j}^{0} \epsilon_{k l}^{0}
$$

This is a problem of maximizing strain energy for a given fixed strain $\epsilon^{0}$, where $C$ ranges over all composites. For periodic composites, given by a cell $Y$ of periodicity, this homogenized strain energy is written as

$$
C_{i j k l}^{H} \epsilon_{i j}^{0} \epsilon_{k l}^{0}=\underset{\phi Y \text {-periodic }}{\min } \int_{\Omega} C_{i j k l}(y)\left(\epsilon_{i j}^{0}-\epsilon_{i j}(\phi)(y)\right)\left(\epsilon_{k l}^{0}-\epsilon_{k l}(\phi)(y)\right) d Y
$$

and the material design problem becomes

$$
\max _{C \in E_{\mathrm{ad}^{(Y)}}} C_{i j k l}^{H} \epsilon_{i j}^{0} \epsilon_{k l}^{0}
$$

where $E_{\text {ad }}$ is denotes the set of admissible local material tensors, or, rather, the local design of the microstructure. For layered materials, this is an analytical problem, but one could also think of this problem as a computational problem of local topology design. Note that problems (8), (10) generate an upper bound on the strain energy of any composite, and thus by choice of strain $\epsilon^{0}$ on the material properties of such composites. An example would be to find bounds on the bulk and shear moduli of isotropic composites.

\section{The SIMP model}

Complementing the use of the homogenization method, where anisotropic composites are a priori accepted as part of the design space, a popular method to model material properties which are isotropic at intermediate densities is the so-called penalized, proportional fictitious material SIMPmodel (SIMP: Solid Isotropic Material with Penalization), see for example [9] for an overview. In this model a continuous variable $\rho$ is introduced, with $0 \leq \rho \leq 1$, resembling a density of material by the fact that the volume of the structure is evaluated as

$$
V=\int_{\Omega} \rho(x) d \Omega
$$

The relation between this density and the material tensor $C_{i j k l}$ in the equilibrium analysis is written as

$$
C_{i j k l}(\rho)=\rho^{p} C_{i j k l}^{0}, \quad p>1,
$$

where the given material has stiffness given by $C_{i j k l}^{0}$. The interpolation (12) satisfies that $C(0)=0$ and $C(1)=C_{i j k l}^{0}$, meaning that if a final design has density zero and one in all points, this design is a black-and-white design for which the performance has been evaluated with a correct physical model. For problems where the volume constraint is active, experience shows that optimization 
does actually result in such designs if one chooses the power $p$ sufficiently big (in order to obtain true ' $0-1$ ' designs, $p \geq 3$ is usually required). The reason is that for such a choice one imposes a penalization on intermediate densities (volume is proportional to $\rho$ but stiffness is less than proportional). The SIMP model thus has an inherent penalization leading to 0-1 designs, and, as such, requires filtering or likewise for existence of solutions (cf., the discussion in the introduction).

For the SIMP interpolation (12) it is not immediately apparent that areas of grey can be interpreted in physical terms. However, it turns out that under fairly simple conditions on $p$, any stiffness used in the SIMP model can be realized as the stiffness of a composite made of void and an amount of the base material corresponding to the relevant density, see Figure 2. Thus using the term 'density' for the interpolation function $\rho$ is quite natural, and this type of model actually also falls under the label 'the homogenization method for topology design'.

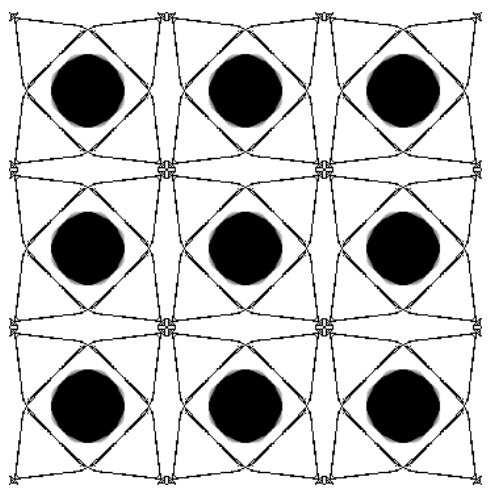

Density 0.25

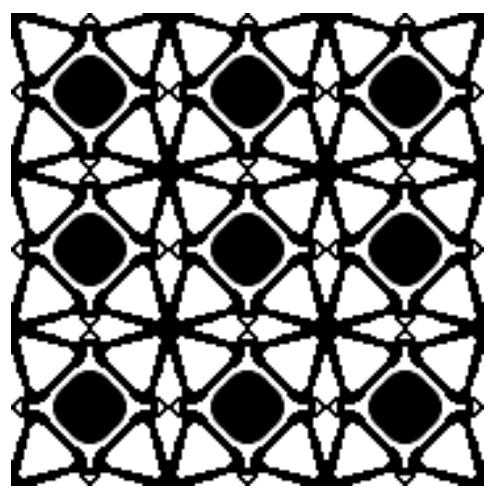

Density 0.5

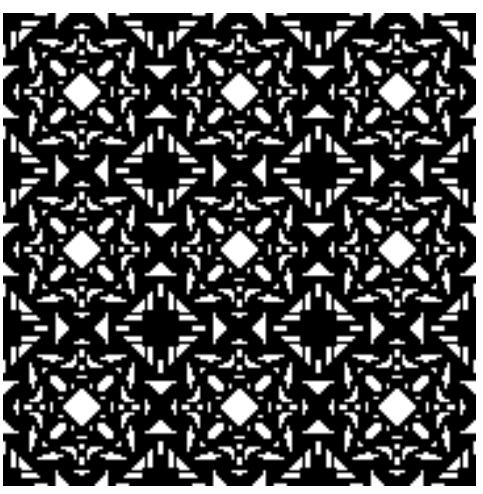

Density 0.75

Figure 2: Planar microstructures of material and void realizing the material properties of the SIMP model with $p=4$ (Poisson's ratio is zero). As stiffer material microstructures can be constructed from the given densities, non-structural areas are seen at the cell centers. See [9].

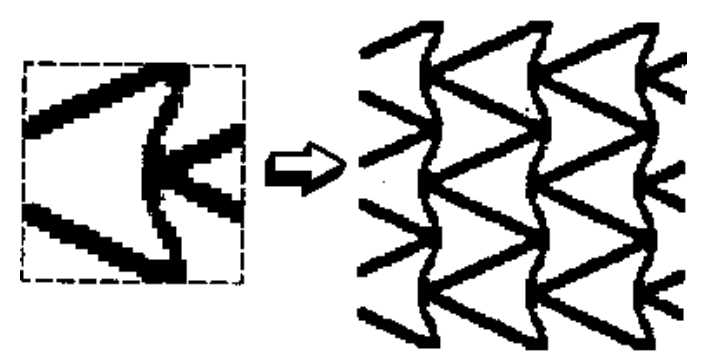

Figure 3: Design of a periodic composite with negative Poisson ratio. Topology optimization is applied to the unit cell of periodicity, and the effective parameters of the composite are computed via homogenization. By courtesy of O. Sigmund, [38]. 


\section{Design of materials}

The basic concept of topology optimization can in itself be used to design material microstructures. The idea is to use expressions of the form (9) for the homogenized material parameters of a periodic composite to state conditions on the resulting material to be designed (e.g., by least squares), and then to use topology design to find the optimal distribution of material in the unit cell, which is now the reference domain. Typically one wish to find single length-scale microstructures, and thus a verison of the SIMP interpolation with geometric constraints (a filter) is a natural version, cf., ([38]). Such a procedure is inverse homogenization. It can result in many interesting types of material microstructures, as for example ones that result in a medium which has homogenized properties with a negative Poisson's ratio, see Figure 3. Also, by this method one can obtain the geometries in Figure 2 ([9]), which are isotropic microstrutures representing the properties of the SIMP interpolation. Thus one has here employed topology design with SIMP to obtain specified material properties of SIMP, making the dog bite its tail.

It is in connection with material design useful to compare the values of the strain energy (c.f., (9)) of the composites and relate this to the known bounds on the effective material parameters of composites. For comparison one gets a clearer picture when considering the dual of this, that is, the complementary energy, i.e.,

$$
\left(\left[C^{H}\right]^{-1}\right)_{i j k l} \sigma_{i j} \sigma_{k l}
$$

for a given stress $\sigma$. If any 2-D anisotropic composite made of void and an isotropic material with Youngs modulus $E^{0}$ and Poisson ratio $\nu^{0}$ is allowed, the upper bound on the stiffness, that is, the lower bound on the complementary energy can for any stress field be found by the material properties of an optimally designed rank-2 layering, with directions of layerings along the directions of the principal stresses. The bound is given by (see, e.g., [3] and references therein):

$$
\left[C^{-1}\right]_{i j k l} \sigma_{i j} \sigma_{k l} \geq \begin{cases}\frac{1}{E^{0} \rho}\left[\sigma_{I}^{2}+\sigma_{I I}^{2}-2\left(1-\rho+\rho \nu^{0}\right) \sigma_{I} \sigma_{I I}\right] & \text { if } \sigma_{I} \sigma_{I I} \leq 0 \\ \frac{1}{E^{0} \rho}\left[\sigma_{I}^{2}+\sigma_{I I}^{2}+2\left(1-\rho-\rho \nu^{0}\right) \sigma_{I} \sigma_{I I}\right] & \text { if } \sigma_{I} \sigma_{I I} \geq 0\end{cases}
$$

for a stress field $\sigma$ with principle stresses $\sigma_{I}$ and $\sigma_{I I}$. This bound has been found by analytical means. It is useful to compare this bound with the corresponding optimal complementary energy which can be obtained with other composites, see Figure 4. It is here especially interesting to note that the numerically obtained single lenth-scale so-called Vidgergauz-like structures ([48]) (see Figure 5) have complementary energy as low as the optimal bound for principal stresses of the same sign, while it does not seem to be possible otherwise. This is confirmed by the recent results ([1]) which show that no single scale periodic composite obtain the bounds in this case, and any composite obtaining the bound (in 2-D) must be degenerate (i.e. has a singular stiffness tensor)

For the SIMP model the bounds to consider are not the ones given in (14), but rather the Hashin-Shtrikman bounds ([20]) on the effective material parameters of isotropic composites, as illustrated graphically in Figure 6 for an isotropic base material with Poisson ratio 1/3 (in this case the bounds can be expressed solely in terms of the Young's modulus). Such an comparison shows that for example in 2-D, the power $p$ of the SIMP model should satisfy

$$
p \geq \max \left\{\frac{4}{1+\nu}, \frac{2}{1-\nu}\right\}
$$




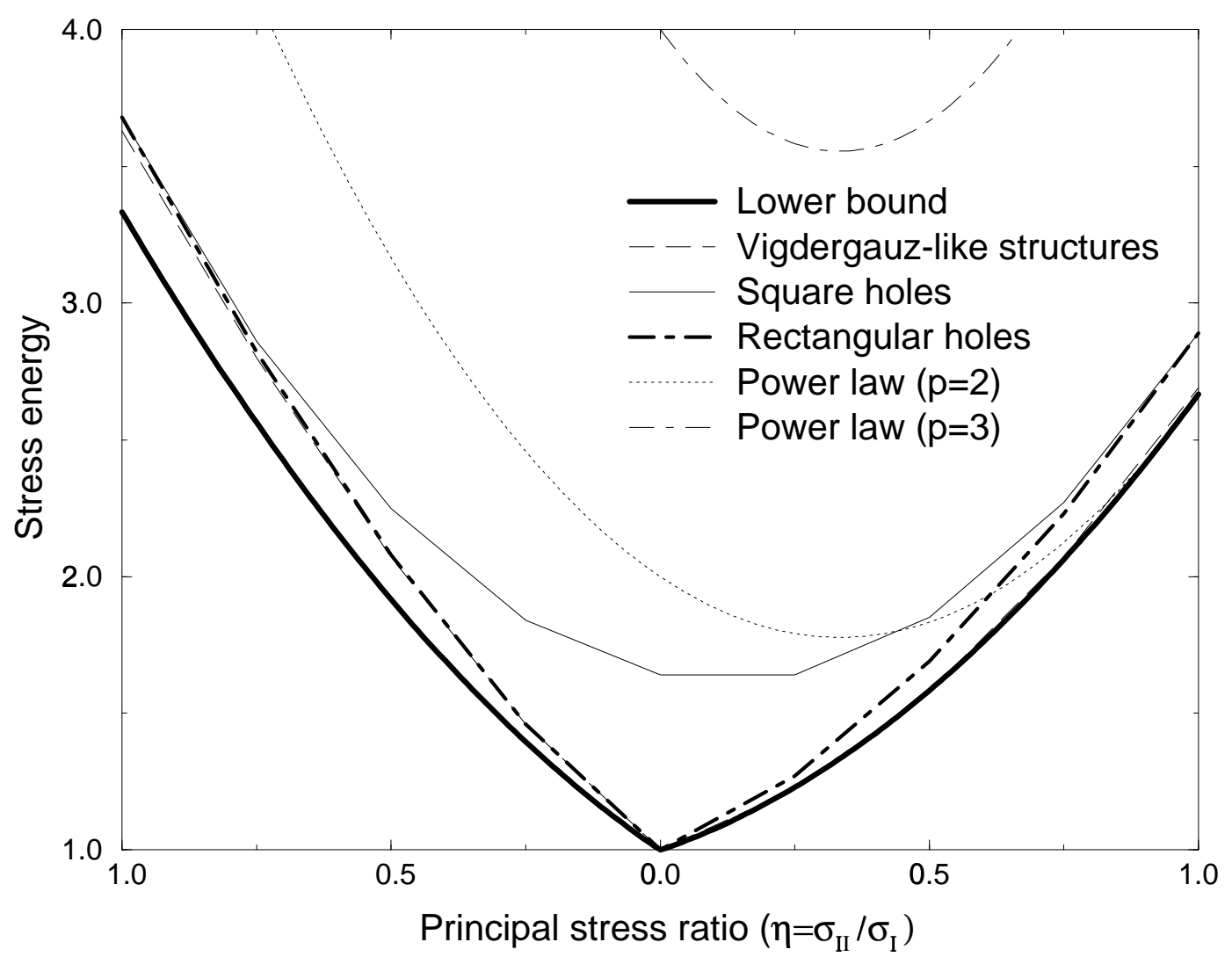

Figure 4: Comparison of the optimal (minimal) complementary energy as a function of the ratio of the principal stresses, and for various types of microstructures and interpolation schemes (material and void mixtures). The density is $\rho=0.5$ and Poisson's ratio is $\nu=1 / 3$. The Vidgergauz-like structures are shown in Figure 5 ([9]). 


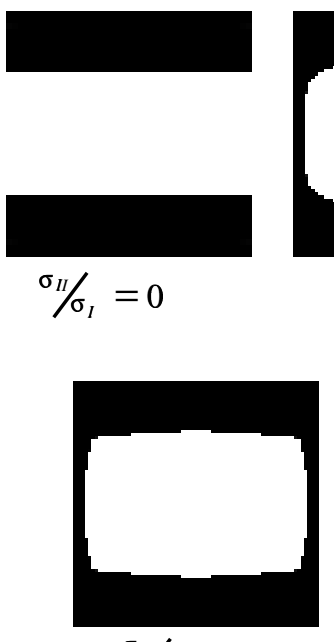

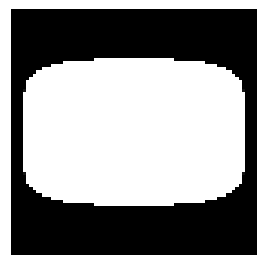

$\sigma_{I I} / \sigma_{I}=0.25$

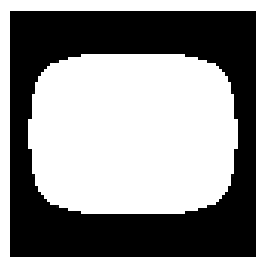

$\sigma_{I I} / \sigma_{I}=0.5$

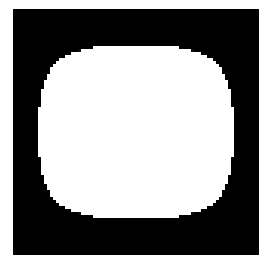

$\sigma_{I I} / \sigma_{I}=0.75$

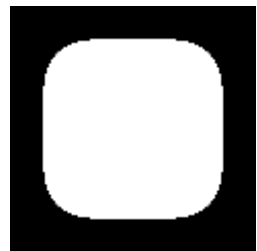

$\sigma_{I I} / \sigma_{I}=1$
$\sigma_{I I} / \sigma_{I}=-0.25$

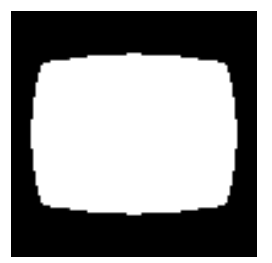

$\sigma_{I I} / \sigma_{I}=-0.5$

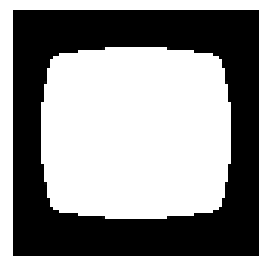

$$
\sigma_{I I} / \sigma_{I}=-0.75
$$

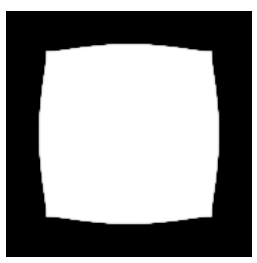

$$
\sigma_{I I} / \sigma_{I}=-1
$$

Figure 5: The shape of single inclusions of void in a cell of a homogenized, periodic medium minimizing complementary energy (Vigdergauz-like structures for $\nu=1 / 3$ and a density $\rho=$ $0.5)$. Results for a range of principal stress ratios of a macroscopic stress field ([9]).

in order for this model to satisfy the Hashin-Shtrikman bounds, i.e., it is a necessary condition for the model to be represented by a composite. Moreover, the material design technique then shows that this is also possible, as illustrated in Figure 2 (see [9] for further details).

An issue arising in topology design of microstructures - as also in topology design in general - is the appearance of slender elements of the structures, often making such structures vulnerable to instability phenomena such as buckling. A global buckling criterion for topology design of continuum structures has been considered in [28], and this development has been used as a basis for designing composites for optimal elastic properties (e.g., maximal bulk modulus), while maintaining a lower limit on the critical instability load of the composite. Such instabilities can have several length-scales, depending on the geometry, dimensions and loading of the medium. For design, one can reasonably choose to use homogenization to obtain the buckling load for periodic buckling modes existing at a length-scale comparable to the cell of periodicity, while accounting for the possibility that modes may exist which involve several cells. Thus the design of the microstructure is performed over one cell, but the buckling analysis (in a linear eigenvalue analysis, using homogenized equations) can involve a modified cell of periodicity involving several of the cells used to described the micro-geometry. In principle this should not be necessary, as this corresponds to a special refined geometry of the unit cell, but the limited geometric resolution of the design description makes such an approach necessary as a safeguard. The design problem involves the standard problems of treating eigenvalue problems in topology design. One is the possible non-smoothness of the objective, the other being the appearance of localized modes in low-density areas, requiring special care to identify the relevant modes ([28], [31] - see also below). Filtering is used in order to control geoemtric complexity. The computational experiments show that the range of microgeometries which attain a high bulk modulus is wide enough to allow 


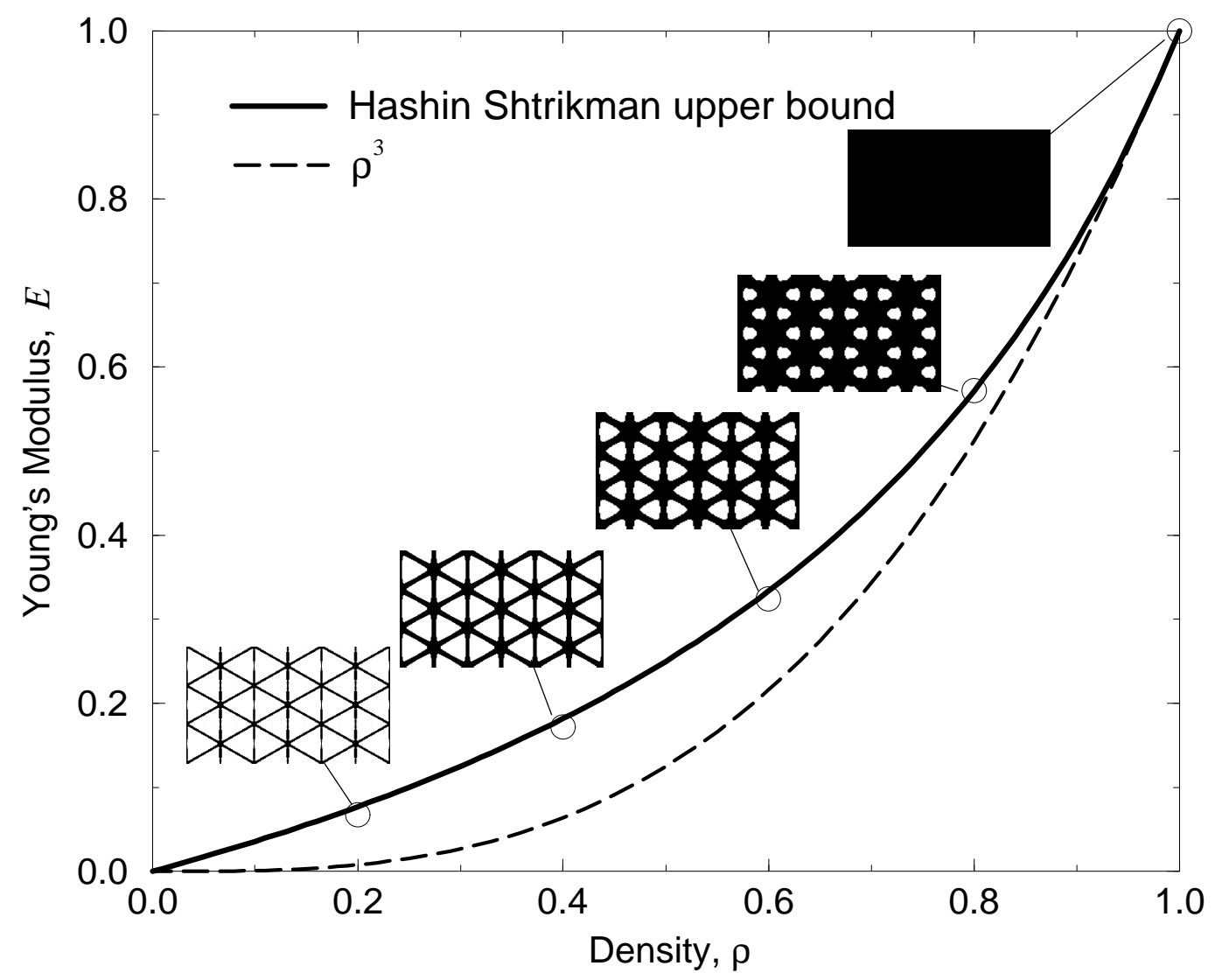

Figure 6: A comparison of the SIMP model and the Hashin-Strikhman upper bound for an isotropic material with Poisson ratio 1/3 mixed with void. For the H-S upper bound, microstructures with properties almost attaining the bounds are also shown ([9]). 
for using microstructures where a reasonable account of the critical load carrying capacity can be taken into account, see Figure 7.

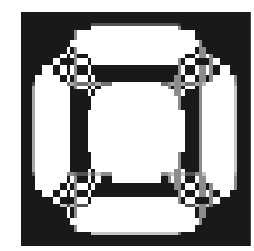

A

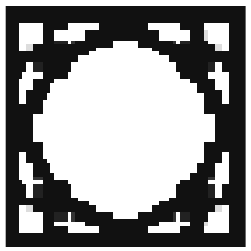

B

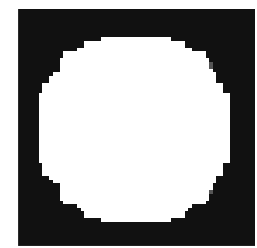

C

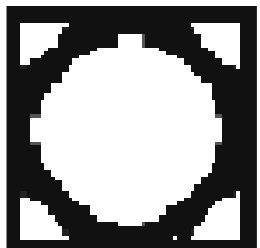

D

Figure 7: Microstructures optimized for optimal bulk properties. For (A) no buckling constraint is imposed, while for (B) such a constraint is used. Note that the buckling constraint also removes checkerboards, as such substructures have a zero numerical buckling load. Finally, in (C) and (D) a filter is used to limit geometric complexity ([27]).

We close this brief discussion on the possibility of designing materials by topology design of the unit cell of a periodic, homogenized medium by noting that the basic concept has been shown to work efficiently for a broad range of problems, also involving for example thermal expansion (see for example [45]) or piezzo-electrical effects (see for example [22]). Moreover, the technique has been instrumental in new work on bounds on the material properties of mixtures of three materials ([18]) as well as for the devlopment of a new class of composites which are useful for exploring the set of material parameters given by the Hashin-Strikhman bounds for two materials ([42]).

\section{Design of mechanisms}

Compliant mechanism plays a significant role for MEMS (Micro Electro Mechanical Systems) applications, as standard techniques for assembly of mechanism is not possible at extremely small scales. On the other hand, small scale allow for the use of new actuation principles, as for example electrically induced heat expansion, where the scale gives a possibility for rapid heating and cooling and thus resonable timescales for movements. Compliant mechanism operate by the flexibility of a structure to perform its task, and the mechanism is thus one structure, for which the basic concept of topology design is applicable, see Figure 8 (see also [39], [29]). For mechanisms the initial topology design studies were carried out using geometrically linear analysis, i.e., small strains were assumed. In practise this is not the case, and moreover it turns out that the geometric linearity actually imposes a restriction on the effectiveness of the mechanisms - for this setting one actually prefers displacement (as opposed to stiffness design). This is illustrated in Figure 9.

Closing this short discussion on mechanism design, we will here briefly outline some aspects of treating multiple physiscs problems. The phrase 'multiple physics' is here used to cover topology design where several physical phenomena are involved in the problem statement, thus covering situations where for example elastic, thermal and electromagnetic analyses are involved. When modelling such situations, the basic concept of the density distribution method for topology 


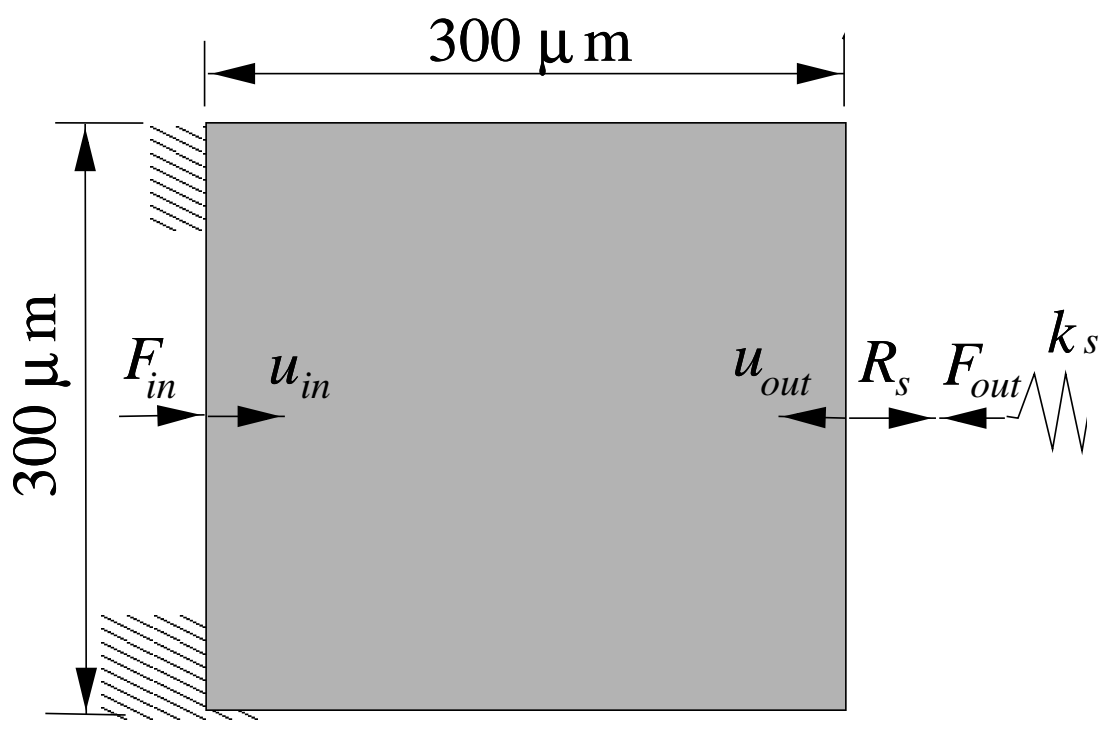

Figure 8: The principle of design of compliant mechanisms. For a certain input force one maximizes the work done on a spring at output. The choice of spring stiffness determines the compromise between output force and output displacement.
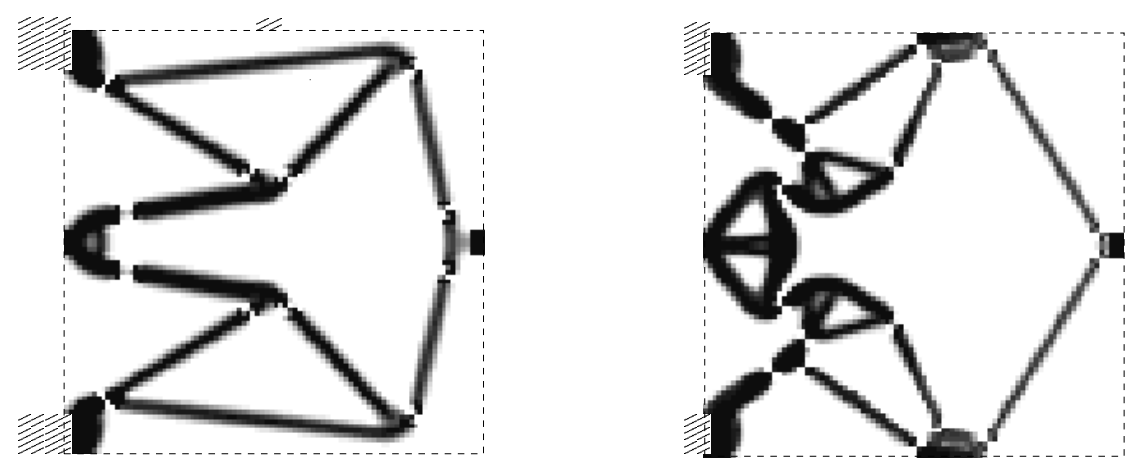

Figure 9: Design of a compliant mechanisms - a force inverter, c.f., the design setting illustrated in Figure 8. The left hand design is designed using geometrically linear analysis: this design will lock for moderately large displacements. The right-hand picture shows a design obtained using geometrically non-linear analysis, and here this allows for substantially larger displacements. By courtesy of B.B.W. Pedersen, T. Buhl and O. Sigmund, [30]. 
design provides a general framework for computations, but here the initial obstacle is the need for interpolation of not only stiffness but also other physical properties. If only linear models are considered, one possibility is to use the theory and computational framework of homogenization of composite media to compute effective elastic, thermal and electromagnetic properties of a given type of composites and use such relationships between intermediate density and material properties in the design problem. An example of this approach for thermo-elastic problems can be found in [35]. However, the less complex design description of the SIMP approach has lead also to the development of such interpolation schemes for multiple physics problems. As an example of this, in reference [45], microstructures with extreme thermal expansion are designed by combining a material interpolation of elastic properties with a similar interpolation of the thermal expansion coefficients. Similar ideas can be used to design mechanisms based on thermo-mechanical and on thermo-electro-mechanical actuation principles, as shown in Figure 10 (see also [40], [41], [43]).

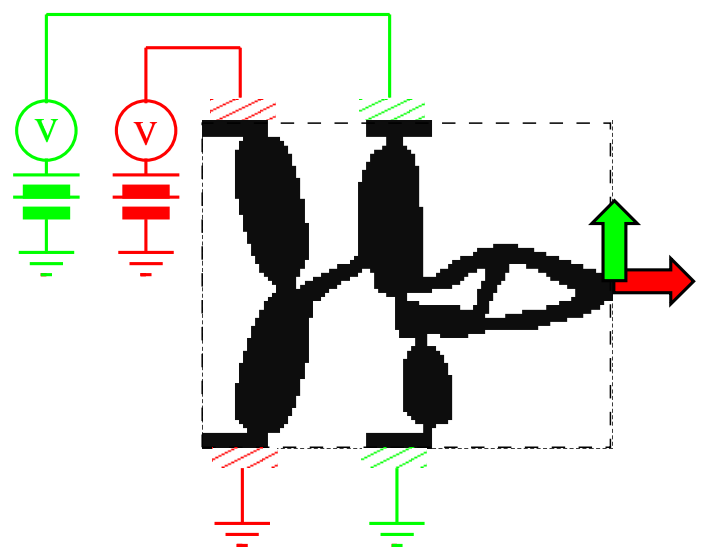

Figure 10: Design of a thermo-electro-mechanical actuator. The mid right-hand point should move right or up when voltage is applied at the corresponding poles. The movement is through elastic deformation due to the temperature change which occurs when a current passes through the structure. By courtesy of O. Sigmund, [40].

\section{Areas of application}

Computational means for topology design have now been developed into reliable tools for problems involving stiffness (including multiple load problems) and vibration criteria. The efficiency of topology design as a tool in the initial phase of a design process and the appearance of commercial software has lead to a broad acceptance of the method in industry (c.f., e.g., [49], [37]; see also www.topopt.dtu.dk for pointers to relevant web-sites). Research has also lead to considering extended classes of problems for example with non-linear material behaviour and local constraints (i.e., constraints imposed on all points in the domain). The exact form of a relaxed form of these problems are in most cases unknown. The emphasis in the field is currently on modelling and on the development of computational means, mostly relying on restriction methods 
as an underlying implicit guarantee of well-posedness. Thus, in the context of theory, the pace of implementations for new problem types has far overtaken the full mathematical understanding of the interplay between methods for obtaining 'classical' solutions and methods for deriving relaxed problem settings, and here lies an immense challenge for the future. For a mathematician there is thus an abundance of (hard) problems to study.

\section{Challenges}

In this presentation models for topology design have been viewed as material distribution problems in a fixed domain. It is central to this concept that computations work with a fixed FEM mesh. This implies that low density areas are also included in the analysis for each feasible design. For stress constraints this leads to the difficulty of the so-called 'stress singularity phenomenon', where low density regions may have high stress but are structurally insignificant for the final design, making computations tricky ([14]). A similarly elusive problem arising from this basic design representation appears in situations involving stability and vibration criteria. The relevant data to consider in such situations are the eigenvalues of the structurally relevant parts of the structure, i.e., the buckling loads and the vibration frequencies of the 'black' part of a black- and-white design. In a true black-and-white design this are the non-zero eigenvalues, but at intermediate steps of an iterative optimization method it can become unclear what are the relevant values to consider for a given grey-scale design. Examples of this are localized modes which appear in low density regions and which should be filtered out in order that the optimization deals with the structurally interesting modes. Moreover, one should cater for certain aspects which complicates modelling, for example that no structure what-so-ever has the highest eigenfrequency of all structures with only structural mass. See references [28] and [31] for further discussion.

For future work it would be beneficial to study alternative approaches to topology design for black-and-white design, perhaps with an emphasis on a geometric modelling along the lines of optimal boundary shape design, as one here have no ambiguity with respect to modelling of material properties and of response. For such work, the concept of the bubble method ([15]) and the concept of a general topological derivative ([46]) could be useful starting points.

\section{WWW}

Much useful information on topology design can be found on the Internet.

At the site www.topopt.dtu . dk one can find tutorials on the subject, a free 99 line MATLAB topology optimization code, a topology optimization test-site allowing you to make your own designs, as well as interesting links to academic and commercial sites.

\section{Acknowledgments}

The author would like to thank O. Sigmund, C.B.W. Pedersen, and T. Buhl for permission to use several of the illustrations shown in this presentation. 


\section{Bibliographie}

[1] G. Allaire and S. Aubry. On optimal microstructures for a plane shape optimization problem. Structural Optimization, 17:86-94, 1999.

[2] G. Allaire, E. Bonnetier, G. Francfort, and F. Jouve. Shape optimization by the homogenization method. Numerische Mathematik, 76:27-68, 1997.

[3] G. Allaire and R.V. Kohn. Optimal bounds on the effective behaviour of a mixture of two well-ordered elastic materials. Quaterly of Appled Mathematics, 51:643-674, 1993.

[4] G. Allaire and R.V. Kohn. Optimal design for minimum weight and compliance in plane stress using extremal microstructures. European Journal of Mechanics A, 12:839-878, 1993.

[5] L. Ambrosio and G. Buttazzo. An optimal design problem with perimeter penalization. Calculus and Variation, 1:55-69, 1993.

[6] M. Beckers. Topology optimization using a dual method with discrete variables. European Journal of Mechanics A, 17:14-24, 1999.

[7] M. P. Bendsøe and N. Kikuchi. Generating optimal topologies in structural design using a homogenization method. Computer Methods in Applied Mechanics and Engineering, 71:197-224, 1988.

[8] M.P. Bendsøe. Optimization of Structural Topology, Shape, and Material. Springer Verlag, Heidelberg, 1995.

[9] M.P. Bendsøe and O. Sigmund. Material interpolation schemes in topology optimization. Archives of Applied Mechanics, 69:635-654, 1999.

[10] B. Bourdin. Filters in topology optimization. Danish Center for Applied Mathematics and Mechanics, DCAMM Report No. 628, December 1999.

[11] G.D. Cheng and N. Olhoff. An investigation concerning optimal design of solid elastic plates. International Journal of Solids and Structures, 17:305-323, 1981.

[12] A. Cherkaev. Variational Methods for Structural Optimization. Springer-Verlag, Berlin / Heidelberg / New York, 2000.

[13] A. Diaz and R. Lipton. Optimal material layout for 3D elastic structures. Structural Optimization, 13:60-64, 1997.

[14] P. Duysinx and M.P. Bendsøe. Topology optimization of continuum structures with local stress constraints. International Journal for Numerical Methods in Engineering, 43:1453$1478,1998$.

ESAIM: Proc., Vol. 11, 2002, 41-60 
[15] H.A. Eschenauer, V. Kobelev, and A. Schumacher. Bubble method of topology and shape optimization of structures. Structural Optimization, 8:42-51, 1994.

[16] C. Fleury. CONLIN: an efficient dual optimizer based on convex approximation concepts. Structural Optimization, 1:81-89, 1989.

[17] G.A. Francfort and F. Murat. Homogenization and optimal bounds in linear elasticity. Arch. Rat. Mech. Anal., 94:307-334, 1986.

[18] L.V. Gibiansky and O. Sigmund. Multiphase composites with extremal bulk modulus. $J$. Mech. and Physics of Solids, 48:461-498, 2000.

[19] J. Goodman, R.V. Kohn, and L. Reyna. Numerical study of a relaxed variational problem from optimal design. Computer Methods in Applied Mechanics and Engineering, 57:107127, 1986.

[20] Z. Hashin and S. Shtrikman. A variational approach to the theory of the elastic behaviour of multiphase materials. J. Mech. Phys. Solids, 11:127-140, 1963.

[21] M.P. Kamat. Structural Optimization -Status and Promise. AIAA, Washington D.C., 1995.

[22] N. Kikuchi, S. Nishiwaki, J.S.O. Fonseca, and E.C.N. Silva. Design optimization method for compliant mechanisms and material microstructure. Computer Methods in Applied Mechanics and Engineering, 151:401-417, 1998.

[23] R.V. Kohn and G. Strang. Optimal design and relaxation of variational problems. Communications in Pure and Applied Mathematics, 39:113-137, 139-182, 353-377, 1986.

[24] K.A. Lurie, A.V. Cherkaev, and A.V. Fedorov. Regularization of optimal design problems for bars and plates, I, II, III. JOTA, 37, 42:499-543, 247-282, 1982.

[25] F. Murat. Contre-exemples pour divers problémes ou le contrôle intervient dans les coefficients. Ann. Mat. Pura ed Appl. Serie 4, 112:49-68, 1977.

[26] F. Murat and L. Tartar. Calcul des variations et homogeneisation, volume Les Methodes de l'Homogeneisation: Theorie et Applications en Physique, pages 319-370. Electricite de France, Eyrolles, Paris, 1985.

[27] M. M. Neves, O. Sigmund, and M. P. Bendsøe. A buckling performance index for topology design of periodic microstructures. In Proc. ECCOMAS 2000 Barcelona 11-14 September 2000, pages 1-12. ECCOMAS, 2000.

[28] M.M. Neves, J.M. Guedes, and H.C. Rodrigues. Generalized topology design of structures with a buckling load criterion. Structural Optimization, 10:71-78, 1995.

[29] S. Nishiwaki, M.I. Frecker, S. Min, and N. Kikuchi. Topology optimization of compliant mechanisms using the homogenization method. International Journal for Numerical Methods in Engineering, 42:535-559, 1998. 
[30] C.B.W. Pedersen, T. Buhl, and O. Sigmund. Topology synthesis of large -displacement compliant mechanisms. Danish Center for Applied Mathematics and Mechanics, DCAMM Report No. 631, 2000.

[31] N. L. Pedersen. Maximization of eigenvalues using topology optimization. Structural Optimization, 2000. to appear - see also DCAMM Report No. 620.

[32] J. Petersson. A finite element analysis of optimal variable thickness sheets. SIAM Journal of Numerical Analysis, 36:1759-1778, 1999.

[33] J. Petersson. Some convergence results in perimeter-controlled topology optimization. Computer Methods in Applied Mechanics and Engineering, 171:123-140, 1999.

[34] J. Petersson and O. Sigmund. Slope constrained topology optimization. International Journal for Numerical Methods in Engineering, 41:1417-1434, 1998.

[35] H.C. Rodrigues and P. Fernandes. A material based model for topology optimization of thermoelastic structures. International Journal for Numerical Methods in Engineering, 38:1951-1965, 1995.

[36] G.I.N. Rozvany. Topology Optimization in Structural Mechanics. Springer Verlag, Heidelberg, 1997.

[37] U. Schramm. The use of structural optimization in automotive design - state of the art and vision. In C. Bloembaum, editor, Proc. Third World Congress of Structural and Multidisciplinary Optimization, pages 200-202. University of New York at Buffalo, 1999.

[38] O. Sigmund. Tailoring materials with prescribed elastic properties. Mechanics of Materials, 20:351-368, 1995.

[39] O. Sigmund. On the design of compliant mechanisms using topology optimization. Mechanics of Structures and Machines, 25:495-526, 1997.

[40] O. Sigmund. Topology optimization in multiphysics problems. In Proc. 7th AIAA/USAF/NASA/ISSMO Symposium on Multidisciplinary Analysis and Optimization St. Louis MI Sept. 2-4 '98, pages 1492-1500. AIAA, 1998.

[41] O. Sigmund. Design of multiphysics actuators using topology optimization, part i, ii. Danish Center for Applied Mathematics and Mechanics, DCAMM Report No. 632, February 2000 .

[42] O. Sigmund. A new class of extremal composites. J. Mech. and Physics of Solids, 48:397$428,2000$.

[43] O. Sigmund and T. Buhl. Design of multiphysics actuators using topology optimization, part iii, large displacements. Danish Center for Applied Mathematics and Mechanics, DCAMM Report No. 632, February 2000. 
[44] O. Sigmund and J. Petersson. Numerical instabilities in topology optimization. Structural Optimization, 16:68-75, 1998.

[45] O. Sigmund and S. Torquato. Design of materials with extreme thermal expansion using a three-phase topology optimization method. Journal of the Mechanics and Physics of Solids, 45:1037-1067, 1997.

[46] J. Sokolowski and A. Zochowski. On the topological derivative in shape optimization. SIAM Journal on Control and Optimization, 37:1251-1272, 1999.

[47] K. Svanberg. The method of moving asymptotes - a new method for structural optimization. International Journal for Numerical Methods in Engineering, 24:359-373, 1987.

[48] S. Vigdergauz. Regular structures with extremal elastic properties. Mechanics of Solids, 24:57-63, 1989.

[49] R.J. Yang and A.I. Chahande. Automotive applications of topology optimization. Structural Optimization, 9:245-249, 1995.

[50] J. Zowe, M. Kocvara, and M.P. Bendsøe. Free material optimization via mathematical programming. Mathematical Programming B, 79:445-466, 1997.

Martin P. Bendsøe

Department of Mathematics

Matematiktorvet

B. 303

Technical University of Denmark

DK-2800 Lyngby, Denmark

mailto: M.P.Bendsoe@mat.tdu.dk

http://www.mat.dtu.dk/persons/Bendsoee_Martin_P 